\title{
Development of FBG Tension and Tilt Sensors Applied to Ice Monitoring of Overhead Transmission Lines
}

\author{
Dengjin Wu \\ North China Electric Power University, Beijing, China, 102206 \\ hunter2011@foxmail.com
}

\begin{abstract}
Keywords: overhead transmission line, tension and tilt sensors, U-type structure of double closed-loop, FBG
\end{abstract}

\begin{abstract}
Overhead icing transmission lines face great danger of stable operation. Based on the existing transmission line monitoring power, susceptible to electromagnetic interference and other shortcomings and Fiber Bragg Grating (FBG) sensor have the advantages of anti-electromagnetic interference, high sensitivity, small volume and so on, we develop a set of FBG sensing technology of transmission line icing monitoring system. In order to improve the existing FBG column type weighing sensor performance, better monitoring of wire icing conditions developed for resistance to a tower of the FBG double closed-loop $U$ type tension sensor, can be a number of important parameters of the measurement of conductor icing thickness, tension, and sag. The FBG dual closed-loop U type tension sensor is composed of two parts, the FBG sensor and the elastic body. In the sensor, through the design of double closed loop U-shaped structure formed a high precision and anti-partial load ability of the force sensing element; elastic force, in the U-shaped groove surface can be accordingly generates the tensile and compressive principal strain, two FBG wavelength value subtraction that can eliminate the cross sensitivity of FBG strain and temperature and without additional compensation measures. Laboratory test results show that the developed fiber Bragg grating sensor force measuring unit high linearity, pull sensing sensitivity for $0.0413 \mathrm{pm} / \mathrm{N}$, resolution of $24.21 \mathrm{~N}$, nonlinear error was $1.7 \%$, accuracy is $2.37 \%$, relative previous FBG column type weighing sensor, nonlinear error decreased by 40\%, the accuracy is improved by 1.2 times. All aspects of performance have improved.
\end{abstract}

\section{Introduction}

Overhead icing transmission lines easily lead to disconnection, insulator flashover, tower collapse accident, causing a great damage to the society. Therefore, the on-line monitoring of the icing state of the overhead transmission line is very important for improving the reliability and safety of the line. FBG is a carrier for transmission and transformation of optical signal, the use of optical fiber transmission signal, compared to the traditional resistance strain gauge sensor, it has the advantages of anti-electromagnetic interference, high sensitivity, small size, etc., so it is suitable for electromagnetic interference and strong, such as bad line environment. At present after previous laboratory research, has developed a FBG column type weighing sensor, but the measurement accuracy is low, the resolution is only $38.5 \mathrm{~N}$ and sensitivity for $0.142 \mathrm{pm} / \mathrm{N}$, nonlinear error is $2.9 \%$, accuracy is $5.24 \%$,. Because of the influence of temperature and strain at the same time, according to the control variable method, the FBG is required to compensate for the temperature of the FBG. In addition, when the transmission line icing is weighed, it is needed to get the information of the axial tension and sag of the wire, and the above information can be measured in the straight line tower. Resistance to a large tension tower, so the combination of angle sensor, in line with the installation of FBG pull angle sensor, through the transmission line icing monitoring, you can get a number of information, such as the wire tension, sag, and so on. We design a novel fiber Bragg grating strain gauge double loop U-shaped structure in order to improve the shortage of the traditional weighing sensor. 


\section{Principles of Fiber Grating Sensor}

FBG has its unique properties as a narrow band reflection filter passive device. Fiber Bragg grating with the photoblastic effect and thermal effect respectively determine the measurement results are affected by external temperature and strain, common effect: when the role of external strain, elastic optic effect makes the fiber Bragg grating effective refraction rate changes, reflected light wavelength will drift; similarly, when the outside temperature changes, the effective refraction rate in thermal effect will change, reflected light wavelength will drift. The relationship between the central wavelength of fiber brag grating reflection wavelength and the temperature is as follows:

Stands for the center wavelength; stands for fiber material thermalcoefficient; zeta for the thermo optic coefficient of the fiber; e fiber valid elastic optic coefficient; for temperature change,stands for strain changes.

\section{FBG Tension and Tilt Sensors}

Main Structure of Tension and Tilt Sensors: U-type Structure of Double Closed-loop. When the sensor havecoated by ice, the tension will change. The FBG tension and tilt sensors can measure the size of the corresponding change.Tension sensor elastomer used alloy steel of 35CrMnSiA. This material has high elastic limit, elastic aftereffect, elastic hysteresis, small, impact resistance and good fatigue resistance and thermal expansion coefficient and other advantages, suitable for production of large range, high precision tension sensor. Experiment, the structure of the head loaded.When pulling force sensing unit of force, the strain is not uniform, by partial load effect, therefore the developed tension sensor on the anti-partial load ability request is higher. The traditional mass range weighing sensor has the structure of a cylindrical shape, a circular plate, and $\mathrm{S}$ type. The two types of plate ring and S shape are suitable for small strain gauges, which are not suitable for the large size of the fiber grating strain gauge, so the cylindrical structure is unable to measure accurately. We design the double closed loop U-shaped structure, combined with the column structure and ring plate structure has the advantages, in applicable to larger volume of fiber Bragg grating strain at the same time, can improve the tension measuring accuracy and the carrying capacity of partial resistance. The real object picture is as follows:

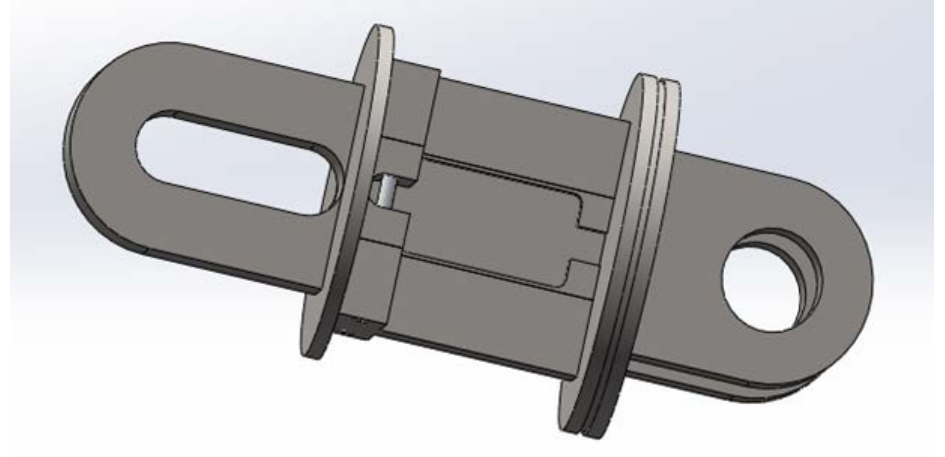

Fig. 1 Structure of Elastomer 


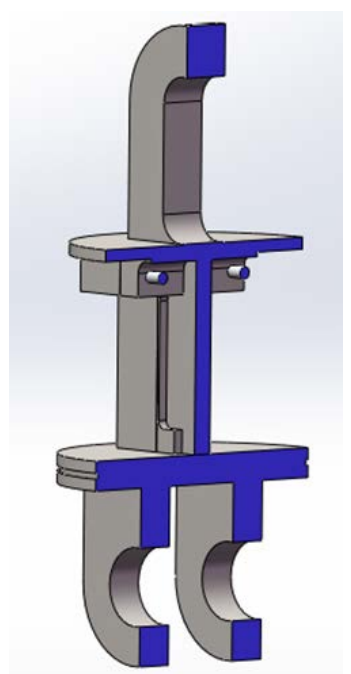

Fig. 2 Cutaway View of Structure of Elastomer

We know the following formula. In the formula, $\mathrm{L}$ is an elastic variable, and $\mathrm{S}$ is the area of stress.

The U-shaped trough cross sectional area is smaller and subjected to tensile deformation, according to the formula shows that the deformation is large, U-shaped groove of the tension sensing unit can more sensitive feelings to deformation, when the tension reaches a certain degree (the critical point of the metal beam effective deformation, depending on metal beam materials used), metal beams will drive on both sides of the web together deformed. Laboratory and metal beam materials used in carbon steel, when the tension is less than $5 \mathrm{kn}$, only $\mathrm{u}$ - tank deformation, when the rally reached $5 \mathrm{kn}$, metal beams reach effective deformation critical point, so as to drive the U-shaped groove on both sides of the web deformation, at this time, elastic body both side by the tension by the U-shaped groove and two side webs bear, stress cross-sectional area s increasing, under the same deformation, can bear large pulling force. Therefore, when the elastic body is less than $5 \mathrm{KN}$, the strain is smaller, and the strain is smaller than $5 \mathrm{KN}$. The effect with practical application, in a normal year, won't appear such as the snowstorm, transmission by wire pulling force is small, high sensitivity can get more accurate data, taking it as reference, used to determine the frequency of inspection and maintenance of transmission line; in the snowstorm of the year, the measuring device is when the transmission line capacity threshold is reached, a reminder was sent. The measurement system of the process curve is shown in the following figure:

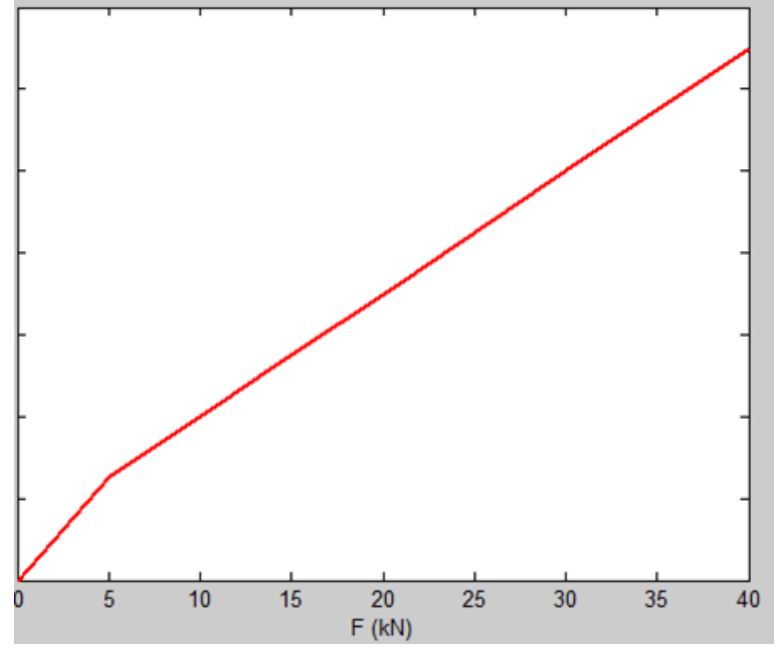

Fig. 3 Relationship between of Elastic Deformation and Tension

Performance Verification of Fiber Grating Sensor. When the section area is $112 \mathrm{~mm}^{2}$, the traditional column is $1052.064 \mathrm{~mm}^{4}$ and the moment of inertia of the double closed loop U-shaped structure elastomer $7189.33 \mathrm{~mm}^{4}$, greatly improve the flexural capacity. At the same time, compared to the traditional column structure, in the double closed loop of a U-shaped elastic body, the fiber Bragg grating strain sheet located by the stress and strain distribution is more uniform, and thus 
measurement accuracy is greatly improved. In the spherical head range of 330 degrees of partial load pull simulation. Through the calculation of finite element calculation software, the corresponding position of the column and double loop U-shaped elastic body strain results such as shown in Fig. 4.

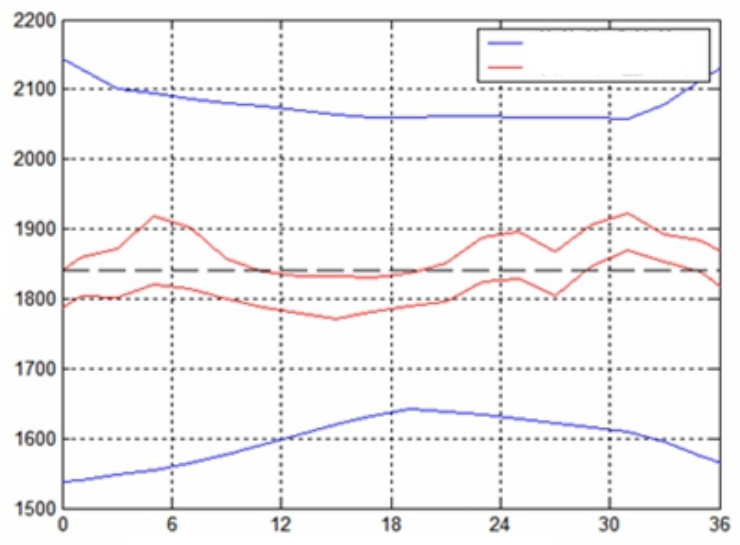

Fig. 4 Simulation Result Comparison of Elastic Strain under Offset Load Condition

Calculation shows that, when the two wavelength variation of FBG1, FBG2, they can eliminate the cross sensitivity of FBG strain and temperature, which does not need extra compensation measures. It is seen that the wavelength change and the elastic body are linearly related to the tensile force. Therefore, the linear change of the wire suspension can be found by measuring the change of the central wavelength of FBG. At the same time, the error can be further reduced by the temperature compensation and dip angle of the sensor, which can improve the accuracy of ice cover monitoring.

\section{Conclusion}

(1) The FBG U-type structure of double closed-loop tension sensor is stressed by tension, the U-shaped groove surface can be accordingly generates the tensile and compressive principal strain. The calculated two FBG wavelength value subtraction, will eliminate the cross sensitivity of FBG temperature and strain of the two variables, without the need for additional compensation measures.

(2)The developed tension and tilt sensor has good performance. Its sensitivity is $0.0413 \mathrm{pm} / \mathrm{N}$ and the resolution is $24.21 \mathrm{~N}$. The nonlinear error of the measurement results is only $1.7 \%$, and the accuracy is $2.37 \%$.

\section{References}

[1] G.M. Ma, C.G. Li, J.T. Quan, Y.C. Cheng, J. Jiang, Meng Chenping, TianXiao, Development of Fiber Bragg Grating Tension \& Tilt Sensor for Icing-monitoring of Overhead Transmission Lines, J., Proceedings of the CSEE, 34(2010)132-138.

[2] JiangJian, Development of FBG Tension and Tilt Sensors Applied to Ice Monitoring of overhead Transmission Lines, D., North China Electric Power University,2011.

[3] Xing Yi, Zeng Yi, Sheng Gehao, On-line monitoring system of overhead transmission line icing based on mechanical measurement, J., Automation of Electric Power Systems, 23(2008)81-85.

[4] W.T. Huang, N.L. Tai, Y. Hu, T.Q. Hu, Study andImplementationofIcing Monitoring Systemof Transmission Lines, Water Resources and Power, 10(2012)157-160. 